\title{
Are nonperturbative AdS vacua possible in bosonic string theory?
}

\author{
Peng Wang, ${ }^{*}$ Houwen $\mathrm{Wu},{ }^{\dagger}$ and Haitang Yang ${ }^{\ddagger}$ \\ College of physics, Sichuan University, Chengdu 610065, China
}

(Received 26 June 2019; published 22 August 2019)

\begin{abstract}
In this paper, following the work of Hohm and Zwiebach [arXiv:1905.06583], we show that in bosonic string theory nonperturbative anti-de Sitter (AdS) vacua could exist with all $\alpha^{\prime}$ corrections included. We also discuss the possibility of the coexistence of nonperturbative dS and AdS vacua.
\end{abstract}

DOI: 10.1103/PhysRevD.100.046016

Whether bosonic string theory permits stable de Sitter (dS) or anti-de Sitter (AdS) vacua is a long-standing unsolved problem. There are conjectures that superstring theory does not have solutions of dS vacua [1-3]. Another conjecture states that there is no stable nonsupersymmetric AdS vacuum with fluxes [4]. Incredibly, by analyzing the nonperturbative properties of the spacetime action of closed string theory (also known as the low-energy effective string theory), Hohm and Zwiebach [5,6] recently showed that nonperturbative dS vacua are possible in bosonic string theory. The most important ingredients in their arguments are $O(d, d)$ symmetry and the classification of all of the $\alpha^{\prime}$ corrections for particular configurations.

It is well known from the work of Meissner and Veneziano [7] in 1991 that, at the zeroth order of $\alpha^{\prime}$, when all fields depend only on time the $D=d+1$-dimensional spacetime action of closed string theory reduces to an $O(d, d)$-invariant reduced action. Soon after that, in Refs. [8,9] Sen extended this result to full string field theory. Specifically, by considering an exact solution of the string field that was independent of $m$-dimensional spacetime coordinates $(m \leq d),{ }^{1}$ Sen proved the following. (i) The space of such solutions has an $O(m, m)$ symmetry. In the language of low-energy effective theory, the reduced action derived from such solutions possesses an $O(m, m)$ symmetry to all orders in $\alpha^{\prime}$. (ii) The $m$ coordinates could be all spacelike or include one timelike coordinate, as explained in Ref. [9]. (iii) In the solution space, inequivalent solutions are connected by nondiagonal $O(m) \otimes O(m)$ transformations $[O(m-1,1) \otimes O(m-1,1)$ if one of the $m$ coordinates is timelike]. (iv) Other generators of $O(m, m)$

\footnotetext{
${ }^{1}$ We concentrate on noncompact configurations here.

pengw@scu.edu.cn

iverwu@scu.edu.cn

"hyanga@scu.edu.cn
}

Published by the American Physical Society under the terms of the Creative Commons Attribution 4.0 International license. Further distribution of this work must maintain attribution to the author(s) and the published article's title, journal citation, and DOI. Funded by SCOAP ${ }^{3}$. outside of the nondiagonal $O(m) \otimes O(m)$ [or $O(m-1,1) \otimes$ $O(m-1,1)]$ generate gauge transformations accompanied by a shift of the dilaton, and thus equivalent solutions. On the other hand, in Ref. [10], from the perspective of $\sigma$ model expansion, since the nilpotency of the BRSToperator $Q$ is not altered by an $O(d, d)$ transformation, it was argued that the $O(d, d)$ symmetry should persist at all orders in $\alpha^{\prime}$ for the reduced action. It is expected that, in terms of the standard fields, the $O(d, d)$ transformations receive higher-order $\alpha^{\prime}$ corrections when introducing higher-derivative terms to the reduced action. For configurations depending only on time, to the first order in $\alpha^{\prime}$, in Ref. [11] Meissner demonstrated that one can trade it with standard $O(d, d)$ transformations in terms of $\alpha^{\prime}$-corrected fields. In the appendix we show that this is also true for configurations that only depend on one spatial coordinate $x$, i.e., the case we study in this paper.

As for the yet unknown higher-order $\alpha^{\prime}$ corrections, some important progress has been made recently using the formalism of double field theory [12-17]. Remarkably, in Refs. [6,16] Hohm and Zwiebach demonstrated that, for cosmological, purely time-dependent configurations, the $O(d, d)$-covariant closed string spacetime action can be expressed in a very simple form. All orders of $\alpha^{\prime}$ corrections do not include the trivial dilaton and can be constructed using even powers of $\partial_{t} \mathcal{S}$, where $\mathcal{S}$ is the spatial part of the generalized metric defined in Eq. (5). This surprising simplification of the $\alpha^{\prime}$ corrections enabled them to discuss the nonperturbative solutions. The most interesting result they obtained is that nonperturbative dS vacua are possible for bosonic string theory $[5,6]$, which possibly provides a cornerstone for the connection between string theory and our real world. In this paper, following their derivations, we show that nonperturbative $\mathrm{AdS}$ vacua are also possible with all $\alpha^{\prime}$ corrections for bosonic string theory.

It is worth noting that we work in the string frame and not the Einstein frame, the same as in Hohm and Zwiebach's work [5,6]. It is still unclear if there could be dS or AdS solutions in the Einstein frame. We address this point at the end of this paper. Another issue is that in order to completely determine the dS/AdS vacua, we still 
need to know all of the $\alpha^{\prime}$ corrections. One of the purposes of this paper is to deny the nonexistence of AdS vacua in bosonic string theory, rather than provide exact solutions.

Moreover, we wish to note that Hohm and Zwiebach solutions and our own only work in the string frame and not the Einstein frame, since when we substitute solutions with a constant dilaton field $\phi$ and Hubble parameter $\bar{H}_{0}$ back into the Einstein frame, $\bar{H}_{0}^{E}$ goes to zero and the metric becomes flat.

For the sake of completeness, let us briefly summarize Hohm and Zwiebach's work on nonperturbative dS vacua. Details can be found in Ref. [6]. To the zeroth order of $\alpha^{\prime}$, the $D=d+1$-dimensional spacetime action of closed string theory is

$I_{0} \equiv \int d^{D} x \sqrt{-g} e^{-2 \phi}\left[R+4\left(\partial_{\mu} \phi\right)^{2}-\frac{1}{12} H_{i j k} H^{i j k}\right]$,

where $g_{\mu \nu}$ is the string metric, $\phi$ is the dilaton, and $H_{i j k}=$ $3 \partial_{[i} b_{j k]}$ is the field strength of the antisymmetric KalbRamond $b_{i j}$ field. For cosmological backgrounds, by choosing the synchronous gauge $g_{t t}=-1, g_{t i}=b_{t \mu}=0$,

$$
\begin{aligned}
g_{\mu \nu} & =\left(\begin{array}{cc}
-1 & 0 \\
0 & G_{i j}(t)
\end{array}\right), \quad b_{\mu \nu}=\left(\begin{array}{cc}
0 & 0 \\
0 & B_{i j}(t)
\end{array}\right), \\
\phi & =\phi(t),
\end{aligned}
$$

and defining the $O(d, d)$ dilaton $\Phi$ as

$$
e^{-\Phi}=\sqrt{g} e^{-2 \phi},
$$

the action can be rewritten as

$$
I_{0}=\int d t e^{-\Phi}\left[-\dot{\Phi}^{2}-\frac{1}{8} \operatorname{Tr}\left(\dot{\mathcal{S}}^{2}\right)\right]
$$

with

$$
\begin{aligned}
& M=\left(\begin{array}{cc}
G^{-1} & -G^{-1} B \\
B G^{-1} & G-B G^{-1} B
\end{array}\right), \\
& \mathcal{S}=\eta M=\left(\begin{array}{cc}
B G^{-1} & G-B G^{-1} B \\
G^{-1} & -G^{-1} B
\end{array}\right),
\end{aligned}
$$

where $M$, a $2 d \times 2 d$ matrix, is the spatial part of the generalized metric $\mathcal{H}$ of double field theory, $\dot{A} \equiv \partial_{t} A$, and $\eta$ is the invariant metric of the $O(d, d)$ group

$$
\eta=\left(\begin{array}{ll}
0 & I \\
I & 0
\end{array}\right)
$$

Noticing that $M$ is symmetric and $\mathcal{S}=\mathcal{S}^{-1}$, this action is manifestly invariant under the $O(d, d)$ transformations

$$
\Phi \rightarrow \Phi, \quad \mathcal{S} \rightarrow \tilde{\mathcal{S}}=\Omega^{T} \mathcal{S} \Omega
$$

where $\Omega$ is a constant matrix, satisfying

$$
\Omega^{T} \eta \Omega=\eta .
$$

If we choose the Friedmann-Lemaitre-Robertson-Walker (FLRW) metric, $G_{i j}=\delta_{i j} a^{2}(t)$, and a vanishing KalbRamond field $B=0$,

$$
d s^{2}=-d t^{2}+a^{2}(t) \delta_{i j} d x^{i} d x^{j},
$$

the matrix $\mathcal{S}$ becomes

$$
\mathcal{S}=\left(\begin{array}{cc}
0 & G \\
G^{-1} & 0
\end{array}\right)
$$

Applying the $O(d) \otimes O(d)$ transformation, we obtain a new solution,

$$
\tilde{\mathcal{S}}=\left(\begin{array}{cc}
0 & G^{-1} \\
G & 0
\end{array}\right),
$$

which implies that the action is invariant under $a(t) \rightarrow a^{-1}(t)$, which is known as scale factor duality in traditional string cosmology. The next step is to include the $\alpha^{\prime}$ corrections. Benefiting from the $O(d, d)$ invariance, the corrections are classified by even powers of $\dot{\mathcal{S}}$ only:

$$
\begin{aligned}
I= & \int d^{D} x \sqrt{-g} e^{-2 \phi}\left(R+4(\partial \phi)^{2}-\frac{1}{12} H^{2}\right. \\
& \left.+\frac{1}{4} \alpha^{\prime}\left(R^{\mu \nu \rho \sigma} R_{\mu \nu \rho \sigma}+\ldots\right)+\alpha^{\prime 2}(\ldots)+\ldots\right) \\
= & \int d t e^{-\Phi}\left(-\dot{\Phi}^{2}+\sum_{k=1}^{\infty}\left(\alpha^{\prime}\right)^{k-1} c_{k} \operatorname{tr}\left(\dot{\mathcal{S}}^{2 k}\right)\right) .
\end{aligned}
$$

Equation (12) is the action for the general background with all $\alpha^{\prime}$ corrections. Equation (13) is the $O(d, d)$ covariant action applied to the metric (9) with $B=0$, where $c_{1}=-\frac{1}{8}$ to recover Eq. (4) and $c_{k \geq 2}$ are undetermined constants. Using the action (13), in Refs. [5,6] Hohm and Zwiebach showed that nonperturbative dS vacua are permitted for infinitely many classes of $c_{k \geq 2}$, namely, $a(t)=e^{H_{0} t}$ with $H_{0} \neq 0$.

Now we want to investigate if nonperturbative AdS vacua are also allowed. To address this question, an appropriate ansatz is crucial. We take the ansatz

$d s^{2}=-a^{2}(x) d t^{2}+d x^{2}+a^{2}(x)\left(d y^{2}+d z^{2}+\ldots\right)$,

whose metric components depend on a single space direction, say, $x$. The dimensionality is still $D=d+1$. As we explained earlier, in Refs. [8,9] Sen proved that the 
reduced action based on such solutions also maintains $O(d, d)$ symmetry to all orders in $\alpha^{\prime}$. The coset for the space of such solutions is $O(d-1,1) \otimes O(d-1,1) / O(d-1,1)$, in contrast to $O(d) \otimes O(d) / O(d)$ for the cosmological solutions (9). In the Appendix we explicitly show that for this ansatz the spacetime action (12) also possesses the standard $O(d, d, R)$ symmetry and can be reduced to

$\bar{I}=-\int d x e^{-\Phi}\left(-\Phi^{\prime 2}+\sum_{k=1}^{\infty}\left(\alpha^{\prime}\right)^{k-1} \bar{c}_{k} \operatorname{tr}\left(\mathcal{M}^{\prime 2 k}\right)\right)$,

where $A^{\prime} \equiv \partial_{x} A$. Note the overall minus sign and the fact that we have a new set of undetermined coefficients $\bar{c}_{k}$ other than the $c_{k}$ 's in Eq. (13). It turns out that

$\bar{c}_{2 k-1}=c_{2 k-1}, \quad \bar{c}_{2 k}=-c_{2 k}, \quad$ for $k=1,2,3 \ldots$

and

$$
\mathcal{M}=\left(\begin{array}{cccc}
0 & 0 & -a^{2}(x) & 0 \\
0 & 0 & 0 & a^{2}(x) \delta_{i j} \\
-a^{-2}(x) & 0 & 0 & 0 \\
0 & a^{-2}(x) \delta_{i j} & 0 & 0
\end{array}\right)
$$

where $i, j=y, z, \ldots$. The equation of motion (EOM) of Eq. (15) can be calculated directly,

$$
\begin{aligned}
\Phi^{\prime \prime}+\frac{1}{2} \bar{H} \bar{f}(\bar{H}) & =0, \\
\frac{d}{d x}\left(e^{-\Phi} \bar{f}(\bar{H})\right) & =0, \\
\left(\Phi^{\prime}\right)^{2}+\bar{g}(\bar{H}) & =0,
\end{aligned}
$$

where

$$
\begin{aligned}
\bar{H}(x) & =\frac{a^{\prime}(x)}{a(x)} \\
\bar{f}(\bar{H}) & =d \sum_{k=1}^{\infty}\left(-\alpha^{\prime}\right)^{k-1} 2^{2(k+1)} k \bar{c}_{k} \bar{H}^{2 k-1}, \\
\bar{g}(\bar{H}) & =d \sum_{k=1}^{\infty}\left(-\alpha^{\prime}\right)^{k-1} 2^{2 k+1}(2 k-1) \bar{c}_{k} \bar{H}^{2 k} .
\end{aligned}
$$

It is easy to see that $\bar{g}^{\prime}(\bar{H})=\bar{H} \bar{f}^{\prime}(\bar{H})$. Note that $\bar{H}(x)$ is not the Hubble parameter since our background is space dependent.

Now, let us check whether there is a solution $a^{2}(x)=$ $e^{2 \bar{H}_{0} x}$ for the EOM (18) such that $d s^{2}=-e^{2 \bar{H}_{0} x} d t^{2}+d x^{2}+e^{2 \bar{H}_{0} x}\left(d y^{2}+d z^{2}+\ldots\right)$.

The scalar curvature of this metric is

$$
R=-D(D-1) \bar{H}_{0}^{2},
$$

which implies that the metric (20) is an AdS background for constant $\bar{H}_{0} \neq 0$. To see this more clearly, we apply the transformation $x \rightarrow-\log \left[\bar{H}_{0} \xi\right] / \bar{H}_{0}$ and recover the familiar Poincaré coordinate

$$
d s^{2}=\frac{1 / \bar{H}_{0}^{2}}{\xi^{2}}\left(-d t^{2}+d \xi^{2}+d y^{2}+d z^{2}+\ldots\right) .
$$

So, we have $\bar{H}_{0}=1 / R_{\text {AdS }}$, consistent with Eq. (21). If we do not include the $\alpha^{\prime}$ corrections, $\bar{f}(\bar{H}) \sim \bar{H}_{0}$ is a constant. From the second equation of Eq. (18), we can figure out that $\Phi$ is a constant. Therefore, to satisfy the first equation of Eq. (18) we must have $\bar{H}_{0}=0$, and thus the metric (20) becomes flat and there is no AdS solution. One thus concludes that there is no $D$-dimensional AdS vacuum without fluxes and $\alpha^{\prime}$ corrections.

Our aim is to search for solutions with constant $\bar{H}_{0} \neq 0$. Considering the effects of $\alpha^{\prime}$ corrections, if there is a nonvanishing $\bar{H}_{0}$ solution, $\bar{f}\left(\bar{H}_{0}\right)$ is a constant and then $\Phi$ is also a constant from the second equation of Eq. (18). Finally, from the first and third equations of Eq. (18), we obtain the condition for a nonvanishing $\bar{H}_{0}$ solution:

$$
\bar{f}\left(\bar{H}_{0}\right)=\bar{g}\left(\bar{H}_{0}\right)=0 .
$$

Let us determine the general form of $\bar{f}(\bar{H})$ with specific choices for $c_{k \geq 2}$ that satisfy the condition (23). Instead of $\bar{f}(\bar{H})$, it is better to consider its integral,

$$
\bar{F}(\bar{H}) \equiv \int_{0}^{\bar{H}} f\left(\bar{H}^{\prime}\right) d \bar{H}^{\prime} .
$$

The condition $\bar{f}\left(\bar{H}_{0}\right)=\bar{g}\left(\bar{H}_{0}\right)=0$ is replaced by

$$
\bar{F}\left(\bar{H}_{0}\right)=\bar{F}^{\prime}\left(\bar{H}_{0}\right)=0 .
$$

It is then easy to understand that the general form

$$
\bar{F}(\bar{H})=-d \bar{H}^{2}\left(1+\sum_{p=1}^{\infty} \bar{d}_{p}\left(\alpha^{\prime}\right)^{p} \bar{H}^{2 p}\right) \prod_{i=1}^{k}\left(1-\left(\frac{\bar{H}}{\bar{H}_{0}^{(i)}}\right)^{2}\right)^{2}
$$

admits $2 k$ solutions of AdS vacua: $\pm \bar{H}_{0}^{(1)}, \ldots, \pm \bar{H}_{0}^{(k)}$, for an arbitrary integer $k>0$. So, the question is: do the coefficients $\bar{c}_{k}$ support the functional form (26)? Although it appears impossible to obtain a definite answer, the bottom line is that $\alpha^{\prime}$ corrections do support the possibility of nonperturbative $\operatorname{AdS}$ vacua. It is worth noting that 
"nonperturbative" here means that we use all $\alpha^{\prime}$ corrections to obtain the solution but not to obtain the solution from the two-derivative equations and then be $\alpha^{\prime}$ corrected. There exists another "stronger" version of "nonperturbative", namely, $\bar{F}(\bar{H})$ cannot be expressed by a series expansion of $\alpha^{\prime}$ [18]. The same scenario occurs for the dS vacua as explained in Refs. [5,6].

However, the real story may be intriguing. As an illustration, let us assume that all orders of $\alpha^{\prime}$ corrections have a very special form that gives

$$
\begin{aligned}
\bar{f}(\bar{H}) & =-\frac{2 d}{\sqrt{\alpha^{\prime}}} \sin \left(\sqrt{\alpha^{\prime}} \bar{H}\right) \\
& =-2 d \sum_{k=1}^{\infty}\left(\alpha^{\prime}\right)^{k-1} \frac{1}{(2 k-1) !} H^{2 k-1} .
\end{aligned}
$$

This functional form is a valid candidate for Eq. (19) for special choices of $\bar{c}_{k \geq 2}$. It includes all orders of $\alpha^{\prime}$ corrections and evidently is nonperturbative. The solutions satisfying the condition $\bar{f}\left(\bar{H}_{0}\right)=0$ are

$$
\sqrt{\alpha^{\prime}} \bar{H}_{0}=2 \pi, 4 \pi, \ldots
$$

It is easy to check that $\bar{g}\left(\bar{H}_{0}\right)=0$ is also satisfied for these solutions, leading to a discrete infinity of AdS vacua. However, note the coefficient relations (16) between dS from Eq. (13) and AdS from Eq. (15): $\bar{c}_{2 k+1}=c_{2 k+1}$, $\bar{c}_{2 k}=-c_{2 k}$. We can immediately see that if $\bar{f}(\bar{H}) \sim$ $\sin \left(\sqrt{\alpha^{\prime}} \bar{H}\right)$ for AdS, then the corresponding function $f(H) \sim \sinh \left(\sqrt{\alpha^{\prime}} H\right)$ in $\mathrm{dS}$, and vice versa. But the $\sin h$ function has no nontrivial zero. So, for the trial function (27), AdS or dS vacua cannot coexist and only one of them survives.

This looks like merely a coincidence since in any case one could use a general form of Eq. (26) to permit AdS or $\mathrm{dS}$ vacua. But we have some reasons to conjecture that by plugging the dS (AdS) metric into the yet unknown infinite $\alpha^{\prime}$ expansion, one could sum the series into an expression including a factor that is very close to the trial function of Eq. (27). ${ }^{2}$ In Ref. [19] we showed that, when expressed in Riemann normal coordinates, the AdS (dS) metric can be expressed in a simple form, which is called the $J$-factor by some mathematicians. To see this explicitly, by considering the nonlinear sigma model of string theory

$$
S=-\frac{1}{4 \pi \alpha^{\prime}} \int_{\Sigma} g_{i j}(X) \partial_{\alpha} X^{i} \partial^{\alpha} X^{j},
$$

we can expand $X^{i}$ at some point $\bar{x}$, say, $X^{i}(\tau, \sigma)=\bar{x}^{i}+$ $\sqrt{\alpha^{\prime} \bigvee^{i}}(\tau, \sigma)$, where the $\mathbb{Y}^{i}$ 's are dimensionless fluctuations.

\footnotetext{
${ }^{2}$ We want to emphasize that the real functional form of $\bar{f}(\bar{H})$ could be more complicated than Eq. (27). We simply use this toy model to discuss the coexistence of nonperturbative dS and AdS vacua.
}

Locally around any point, one can always pick Riemann normal coordinates

$$
\begin{aligned}
g_{i j}(X)= & \eta_{i j}+\frac{\ell_{s}^{2}}{3} R_{i k l j} \mathbb{Y}^{k} \bigvee^{l}+\frac{\ell_{s}^{3}}{6} D_{k} R_{i l m j} \mathbb{Y}^{k} \mathbb{Y}^{l} \mathbb{Y}^{m} \\
& +\frac{\ell_{s}^{4}}{20}\left(D_{k} D_{l} R_{i m n j}+\frac{8}{9} R_{i k l p} R_{m n j}^{p}\right) \mathbb{Y}^{k} \mathbb{Y}^{l} \mathbb{Y}^{m} \mathbb{Y}^{n}+\ldots
\end{aligned}
$$

When the background is maximally symmetric, the expansion is greatly simplified and can be summed into a closed form. For dS, we have

$$
\begin{aligned}
S_{d S} & =-\frac{1}{4 \pi} \int_{\Sigma} \partial \mathbb{Y}^{i} \partial \mathbb{Y}^{j}\left[\frac{\sin ^{2}\left(\frac{\sqrt{\alpha^{\prime}}}{R_{d S}} \mathbb{W}\right)}{\left(\frac{\sqrt{\alpha^{\prime}}}{R_{d S}} \mathbb{W}^{2}\right.}\right]_{i}^{a} \eta_{a j}, \\
\left(\mathbb{W}^{2}\right)^{a}{ }_{b} & \equiv \delta_{b}^{a} \mathbb{Y}^{2}-\mathbb{Y}^{a} \mathbb{Y}_{b} .
\end{aligned}
$$

If the background is AdS, we get

$$
\begin{aligned}
S_{\mathrm{AdS}} & =-\frac{1}{4 \pi} \int_{\Sigma} \partial \mathbb{Y}^{i} \partial \mathbb{Y}^{j}\left[\frac{\sinh ^{2}\left(\frac{\sqrt{\alpha^{\prime}}}{R_{\mathrm{AdS}}} \mathbb{W}\right)}{\left(\frac{\sqrt{\alpha^{\prime}}}{R_{\mathrm{AdS}}} \mathbb{W}^{2}\right.}\right]_{i}^{a} \eta_{a j}, \\
\left(\mathbb{W}^{2}\right)^{a}{ }_{b} & \equiv \delta_{b}^{a} \mathbb{Y}^{2}-\bigvee^{a} \mathbb{\mho}_{b} .
\end{aligned}
$$

Noting that $H_{0} \sim 1 / R_{d S}$ and $\bar{H}_{0} \sim 1 / R_{\text {AdS }}$, the results strongly suggest that the beta functions or EOMs of these two actions $S_{d S}$ and $S_{\mathrm{AdS}}$ may behave very similarly to $f(H) \sim \sin \left(\sqrt{\alpha^{\prime}} H\right)$ and $\bar{f}(\bar{H}) \sim \sinh \left(\sqrt{\alpha^{\prime}} \bar{H}\right)$, or, equivalently speaking, there are nonperturbative dS vacua but not nonperturbative AdS vacua, or vice versa. So it looks like we still need more information about the $\alpha^{\prime}$ corrections to give a definite answer.

Finally, we wish to remark that we have only considered the string metric. The relation between the Einstein metric $g_{\mu \nu}^{E}$ and string metric $g_{\mu \nu}$ is $g_{\mu \nu}^{E}=e^{-\frac{4 \phi}{D-2}} g_{\mu \nu}$. When we substitute our solution with a constant $\phi$ and $\bar{H}_{0}=0$ back into the Einstein frame, $\bar{H}_{0}^{E}$ goes to zero and metric the becomes flat. This implies that there is no dS or AdS vacuum when $\phi$ is a constant in the Einstein frame without $\alpha^{\prime}$ corrections.

\section{ACKNOWLEDGMENTS}

We are deeply indebted to Olaf Hohm and Barton Zwiebach for illuminating discussions and advice. We are also grateful to Hiroaki Nakajima, Bo Ning, and Shuxuan Ying for very helpful discussions and suggestions. This work is supported in part by the National Natural Science Foundation of China (Grants No. 11875196, 11375121, and 11005016). 


\section{APPENDIX}

This appendix has to purposes. The first is to explicitly show that, at the leading order in $\alpha^{\prime}$, for our ansatz (14) the $O(d, d)$ symmetry of the spacetime action can be expressed in the standard form in terms of $\alpha^{\prime}$-corrected fields. The derivations follow the same pattern as the calculations in Refs. [7,11], except for some minus signs in particular places that account for the difference between time and space coordinates.

The second purpose is to briefly demonstrate that, based on our ansatz, the closed string spacetime action reduces to Eqs. (15)-(16). The derivations are completely parallel to those in Ref. [6]. Extra minus signs show up in the coefficients $c_{k}$ of the $\alpha^{\prime}$ expansion.

\section{Zeroth order of $\alpha^{\prime}$}

We start with the tree-level closed string spacetime action without $\alpha^{\prime}$ corrections,

$I_{0} \equiv \int d^{D} x \sqrt{-g} e^{-2 \phi}\left[R+4\left(\partial_{\mu} \phi\right)^{2}-\frac{1}{12} H_{i j k} H^{i j k}\right]$,

where $g_{\mu \nu}$ is the string metric, $\phi$ is the dilaton, and $H_{i j k}=$ $3 \partial_{[i} b_{j k]}$ is the field strength of the antisymmetric KalbRamond field $b_{i j}$. The ansatz we use is

$d s^{2}=-a^{2}(x) d t^{2}+d x^{2}+a^{2}(x)\left(d y^{2}+d z^{2}+\ldots\right)$,

$b_{x \mu}=0$,

or

$$
\begin{aligned}
g_{\mu \nu} & =\left(\begin{array}{ccc}
-a^{2}(x) & 0 & 0 \\
0 & 1 & 0 \\
0 & 0 & a^{2}(x) \delta_{a b}
\end{array}\right), \\
b_{\mu \nu} & =\left(\begin{array}{ccc}
0 & 0 & b_{0 b}(x) \\
0 & 0 & 0 \\
b_{a 0}(x) & 0 & b_{a b}(x)
\end{array}\right), \\
\phi & =\phi(x),
\end{aligned}
$$

where $a, b=2,3, \ldots$ Mimicking the metric of cosmological backgrounds, we choose $b_{x \mu}=0$ in our ansatz. It turns out that this gauge is crucial to preserving the $O(d, d)$ symmetry. In order to obtain the reduced action by using the ansatz (A3), we rotate between the time-like $t$ and the first space-like $x$ directions and rewrite the metric and $b_{\mu \nu}$ as

$g_{\mu \nu}=\left(\begin{array}{cc}1 & 0 \\ 0 & G_{i j}(x)\end{array}\right), \quad b_{\mu \nu}=\left(\begin{array}{cc}0 & 0 \\ 0 & B_{i j}(x)\end{array}\right)$,

where

$$
\begin{aligned}
G_{i j}(x) & \equiv\left(\begin{array}{cc}
-a^{2}(x) & 0 \\
0 & a^{2}(x) \delta_{a b}
\end{array}\right), \\
B_{i j}(x) & \equiv\left(\begin{array}{cc}
0 & b_{0 b}(x) \\
b_{a 0}(x) & b_{a b}(x)
\end{array}\right) .
\end{aligned}
$$

So $\quad g_{00} \equiv g_{x x}, \quad g_{11} \equiv g_{t t} \quad$ and $\quad b_{00} \equiv b_{x x}, \quad b_{11} \equiv b_{t t}$. Henceforth, we will use Eqs. (A4) and (A5) as the definitions for $g_{\mu \nu}$ and $b_{\mu \nu}$.

Since we only need to use $G_{i j}$ as a whole to discuss the $O(d, d)$ symmetry and not its components, the time-like minus sign $G_{11}(x)=-a^{2}(x)$ does not show up until we calculate the reduced action. Straightforwardly, the Ricci tensor is

$$
\begin{aligned}
R_{x}{ }^{x}= & -\frac{1}{4} \operatorname{Tr}\left(G^{-1} G^{\prime}\right)^{2}-\frac{1}{2} \operatorname{Tr}\left(G^{-1} G^{\prime \prime}\right)-\frac{1}{2} \operatorname{Tr}\left(G^{\prime-1} G^{\prime}\right), \\
R_{t}{ }^{t}= & -\frac{1}{2}\left(G^{-1} G^{\prime \prime}\right)_{t}{ }^{t}-\frac{1}{4}\left(G^{-1} G^{\prime}\right)_{t}{ }^{t} \operatorname{Tr}\left(G^{-1} G^{\prime}\right) \\
& +\frac{1}{2}\left(G^{-1} G^{\prime} G^{-1} G^{\prime}\right)_{t}{ }^{t}, \\
R_{a}{ }^{b}= & -\frac{1}{2}\left(G^{-1} G^{\prime \prime}\right)_{a}{ }^{b}-\frac{1}{4}\left(G^{-1} G^{\prime}\right)_{a}{ }^{b} \operatorname{Tr}\left(G^{-1} G^{\prime}\right) \\
& +\frac{1}{2}\left(G^{-1} G^{\prime} G^{-1} G^{\prime}\right)_{a}{ }^{b},
\end{aligned}
$$

and

$$
\begin{aligned}
H_{\mu \nu \alpha} H^{\mu \nu \alpha} & =3 H_{0 i j} H^{0 i j}=3 B_{i j}^{\prime}\left(G^{-1} B^{\prime} G^{-1}\right)^{i j} \\
& =-3 \operatorname{Tr}\left(G^{-1} B^{\prime}\right)^{2},
\end{aligned}
$$

where we used the notation

$$
G^{\prime-1} \equiv \frac{d}{d x}\left(G^{-1}\right), \quad \operatorname{Tr}\left(G^{-1} G^{\prime}\right)=g^{\mu \nu} g_{\mu \nu}^{\prime} .
$$

We then introduce the $O(d, d)$-invariant dilaton $\Phi$, defined as

$$
\begin{aligned}
\Phi & \equiv 2 \phi-\frac{1}{2} \ln \left|\operatorname{det} g_{\mu \nu}\right|, \\
\Phi^{\prime} & =2 \phi^{\prime}-\frac{1}{2} \operatorname{Tr}\left(G^{-1} G^{\prime}\right) .
\end{aligned}
$$

Therefore, the action (A1) can be rewritten as

$$
\begin{aligned}
\bar{I}_{0}= & \int d x e^{-\Phi}\left[\Phi^{\prime 2}+\frac{1}{4} \operatorname{Tr}\left(G^{-1} G^{\prime}\right)^{2}-\frac{1}{2} \operatorname{Tr}\left(G^{\prime-1} G^{\prime}\right)\right. \\
& \left.+\frac{1}{4} \operatorname{Tr}\left(G^{-1} B^{\prime}\right)-\operatorname{Tr}\left(G^{-1} G^{\prime \prime}\right)+\Phi^{\prime} \operatorname{Tr}\left(G^{-1} G^{\prime}\right)\right],
\end{aligned}
$$

where we replaced $I_{0}$ with $\bar{I}_{0}$ to indicate that we are working with the ansatz (A2). Using integration by parts, 


$$
\begin{aligned}
\frac{d}{d x}\left[e^{-\Phi} \operatorname{Tr}\left(G^{-1} G^{\prime}\right)\right]= & e^{-\Phi}\left[\operatorname{Tr}\left(G^{-1} G^{\prime \prime}\right)+\operatorname{Tr}\left(G^{\prime-1} G^{\prime}\right)\right. \\
& \left.-\Phi^{\prime} \operatorname{Tr}\left(G^{-1} G^{\prime}\right)\right],
\end{aligned}
$$

the action becomes

$$
\bar{I}_{0}=\int d x e^{-\Phi}\left[\Phi^{\prime 2}-\frac{1}{4} \operatorname{Tr}\left(G^{-1} G^{\prime}\right)^{2}+\frac{1}{4} \operatorname{Tr}\left(G^{-1} B^{\prime}\right)^{2}\right] .
$$

Moreover, we want to point out the sign differences between our ansatz (A2) and the time-dependent FLRW metric:

$$
\begin{aligned}
\operatorname{sign}\left[R_{x x}\right] & =\operatorname{sign}\left[\tilde{R}_{t t}\right], \\
\operatorname{sign}\left[R_{t t}\right] & =-\operatorname{sign}\left[\tilde{R}_{x x}\right], \\
\operatorname{sign}\left[R_{a b}\right] & =-\operatorname{sign}\left[\tilde{R}_{a b}\right], \\
\operatorname{sign}\left[H^{2}\right] & =-\operatorname{sign}\left[\tilde{H}^{2}\right], \\
\operatorname{sign}\left[H_{\mu \nu}^{2}\right] & =-\operatorname{sign}\left[\tilde{H}_{\mu \nu}^{2}\right], \\
\operatorname{sign}\left[(\partial \phi)^{2}\right] & =-\operatorname{sign}\left[(\partial \tilde{\phi})^{2}\right],
\end{aligned}
$$

where $\tilde{A}$ represents the quantities calculated in the timedependent FLRW background. For the Ricci scalar, we have

$$
\operatorname{sign}[R]=-\operatorname{sign}[\tilde{R}]
$$

Finally, the tree-level action (A1) becomes

$$
\bar{I}_{0}=\int d x e^{-\Phi}\left[\Phi^{\prime 2}+\frac{1}{8} \operatorname{Tr}\left(M^{\prime} \eta\right)^{2}\right]
$$

where

$$
M=\left(\begin{array}{cc}
G^{-1} & -G^{-1} B \\
B G^{-1} & G-B G^{-1} B
\end{array}\right), \quad \eta=\left(\begin{array}{cc}
0 & I \\
I & 0
\end{array}\right) .
$$

Since $\operatorname{Tr}\left(M^{\prime} \eta\right)^{2}=\operatorname{Tr}\left(M^{\prime} M^{\prime-1}\right)$, it is easy to see that the tree-level action (A16) is invariant under $O(d, d, R)$ transformations,

$$
\Phi \rightarrow \Phi, \quad M \rightarrow \tilde{M}=\Omega^{T} M \Omega,
$$

where $\Omega$ satisfies $\Omega^{T} \eta \Omega=\eta$. Considering the gravitational background with $B_{i j}=0$ and a global transformation by $\eta \in O(d, d, R)$, we have
$M=\left(\begin{array}{cc}G^{-1} & 0 \\ 0 & G\end{array}\right) \rightarrow \tilde{M}=\eta M \eta=\left(\begin{array}{cc}G & 0 \\ 0 & G^{-1}\end{array}\right)$.

This is the space-dependent duality corresponding to the scale-factor duality in the time-dependent FLRW background.

\section{First-order correction of $\boldsymbol{\alpha}^{\prime}$}

We now demonstrate that the closed string spacetime action with the first-order $\alpha^{\prime}$ correction also possesses the standard $O(d, d)$ symmetry for our ansatz (A2). The action with the first-order $\alpha^{\prime}$ correction and vanishing KalbRamond field is

$$
\begin{aligned}
I= & \int d^{D} x \sqrt{-g} e^{-2 \phi}\left[R+4\left(\partial_{\mu} \phi\right)^{2}\right. \\
& \left.-\alpha^{\prime} \lambda_{0} R_{\mu \nu \sigma \rho} R^{\mu \nu \sigma \rho}+\mathcal{O}\left(\alpha^{\prime 2}\right)\right] .
\end{aligned}
$$

With some $\alpha^{\prime}$-corrected field redefinitions as in Ref. [11], it can be expressed with first-order derivatives as

$$
\begin{aligned}
I= & \int d^{D} x \sqrt{-g} e^{-2 \phi}\left[R+4\left(\partial_{\mu} \phi\right)^{2}\right]-\alpha^{\prime} \lambda_{0} \int d^{D} x \sqrt{-g} e^{-2 \phi} \\
& \times\left[-R_{G B}^{2}+16\left(R^{\mu \nu}-\frac{1}{2} g^{\mu \nu} R\right) \partial_{\mu} \phi \partial_{\nu} \phi\right. \\
& \left.-16 \partial^{2} \phi(\partial \phi)^{2}+16(\partial \phi)^{4}\right]+\mathcal{O}\left(\alpha^{2}\right),
\end{aligned}
$$

where $R_{G B}^{2}$ is the Gauss-Bonnet term:

$$
R_{G B}^{2}=R_{\mu \nu \sigma \rho} R^{\mu \nu \sigma \rho}-4 R_{\mu \nu} R^{\mu \nu}+R^{2} .
$$

By using our ansatz (A2), as one can check directly, the action turns out to be

$$
\begin{aligned}
\bar{I}= & -\int d x e^{-\Phi}\left\{-\Phi^{\prime 2}-\frac{1}{8} \operatorname{Tr} \mathcal{M}^{\prime 2}+\alpha^{\prime} \lambda_{0}\right. \\
& \left.\times\left[\frac{1}{16} \operatorname{Tr} \mathcal{M}^{\prime 4}-\frac{1}{64}\left(\operatorname{Tr} \mathcal{M}^{\prime 2}\right)^{2}-\frac{1}{4} \Phi^{\prime 2} \operatorname{Tr} \mathcal{M}^{\prime 2}-\frac{1}{3} \Phi^{\prime 4}\right]\right\} \\
& +\mathcal{O}\left(\alpha^{\prime 2}\right),
\end{aligned}
$$

with

$$
\mathcal{M} \equiv M \eta=\left(\begin{array}{cc}
0 & G^{-1} \\
G & 0
\end{array}\right) .
$$

Now, let us introduce the Kalb-Ramond field to the action with the first-order $\alpha^{\prime}$ correction, which is given by 


$$
\begin{aligned}
I= & \int d^{D} x \sqrt{-g} e^{-2 \phi}\left[R+4\left(\partial_{\mu} \phi\right)^{2}-\frac{1}{12} H^{2}\right] \\
& -\alpha^{\prime} \lambda_{0} \int d^{D} x \sqrt{-g} e^{-2 \phi}\left[-R_{G B}^{2}+16\left(R^{\mu \nu}-\frac{1}{2} g^{\mu \nu} R\right) \partial_{\mu} \phi \partial_{\nu} \phi-16 \partial^{2} \phi(\partial \phi)^{2}+16(\partial \phi)^{4}\right. \\
& +\frac{1}{2}\left(R^{\mu \nu \sigma \rho} H_{\mu \nu \alpha} H_{\sigma \rho}^{\alpha}-2 R^{\mu \nu} H_{\mu \nu}^{2}+\frac{1}{3} R H^{2}\right)-2\left(D^{\mu} \partial^{\nu} \phi H_{\mu \nu}^{2}-\frac{1}{3} \partial^{2} \phi H^{2}\right) \\
& \left.-\frac{2}{3} H^{2}(\partial \phi)^{2}-\frac{1}{24} H_{\mu \nu \lambda} H_{\rho \alpha}^{\nu} H^{\rho \sigma \lambda} H_{\sigma}^{\mu \alpha}+\frac{1}{8} H_{\mu \nu}^{2} H^{2 \mu \nu}-\frac{1}{144}\left(H^{2}\right)^{2}\right]+\mathcal{O}\left(\alpha^{\prime 2}\right) .
\end{aligned}
$$

Using Eqs. (A4), (A5), (A6), (A7), and (A9), the action above can be expressed in the $O(d, d)$-invariant form (A23), but with

$$
\mathcal{M}=\left(\begin{array}{cc}
B G^{-1} & G-B G^{-1} B \\
G^{-1} & -G^{-1} B
\end{array}\right)
$$

as one can verify by applying the EOM of $\mathcal{M}$ and $\phi$.

The action (A23) can be further simplified by using the tree-level EOM of $\Phi$ from Eq. (A16), which is

$$
\Phi^{\prime 2}+\frac{1}{8} \operatorname{Tr}\left(M^{\prime} \eta\right)^{2}=0 \rightarrow \Phi^{\prime 2}=-\frac{1}{8} \operatorname{Tr} \mathcal{M}^{\prime 2}
$$

Then, the action is reduced to

$$
\begin{aligned}
\bar{I}= & -\int d x e^{-\Phi}\left\{-\Phi^{\prime 2}-\frac{1}{8} \operatorname{Tr} \mathcal{M}^{\prime 2}\right. \\
& \left.+\alpha^{\prime} \lambda_{0}\left[\frac{1}{16} \operatorname{Tr} \mathcal{M}^{\prime 4}+\frac{1}{96}\left(\operatorname{Tr} \mathcal{M}^{\prime 2}\right)^{2}\right]\right\} .
\end{aligned}
$$

This action manifests the invariance under the $O(d, d, R)$ transformation

$$
\Phi \rightarrow \Phi, \quad M \rightarrow \tilde{M}=\Omega^{T} M \Omega
$$

\section{Higher-order corrections of $\boldsymbol{\alpha}^{\prime}$}

For our ansatz, we have shown that the zeroth order and the first order in $\alpha^{\prime}$ can be rewritten in a standard $O(d, d)$ invariant form. Following the same logic as Refs. [5,6], it is reasonable to assume that this is also true for all orders in $\alpha^{\prime}$.

Following the derivations in Ref. [6], we now show that for our ansatz the action can be put into the reduced form (15). From the definition of $\mathcal{M}$, it is easy to get

$$
\operatorname{Tr} \mathcal{M}=\operatorname{Tr} \mathcal{M}^{\prime}=\operatorname{Tr} \mathcal{M}^{\prime \prime}=0
$$

Moreover, $\mathcal{M M}=1$ leads to $\mathcal{M} \mathcal{M}^{\prime}+\mathcal{M}^{\prime} \mathcal{M}=0$, and then

$$
\begin{gathered}
2 \mathcal{M}^{\prime} \mathcal{M}^{\prime}+\mathcal{M}^{\prime \prime} \mathcal{M}+\mathcal{M} \mathcal{M}^{\prime \prime}=0 \quad \text { and } \\
\mathcal{M} \mathcal{M}^{\prime 2 k+1}=-\mathcal{M}^{\prime 2 k+1} \mathcal{M}
\end{gathered}
$$

Multiplying by $\left(\mathcal{M}^{\prime}\right)^{2 k+1}$ and taking traces, one finds

$$
\operatorname{Tr}\left(\mathcal{M}^{\prime 2 k+1}\right)=0, \quad k=0,1, \ldots
$$

Second, by using the equations of motion of the action (A16), higher space-dependent derivatives of $\mathcal{M}$ can be written in the terms of $\mathcal{M}$ and $\mathcal{M}^{\prime}$. Therefore, higher-order corrections of $\alpha^{\prime}$ can be built from $\mathcal{M}$ and $\mathcal{M}^{\prime}$. Third, if the terms of the higher-order $\alpha^{\prime}$ corrections take the form $\operatorname{Tr}\left(\mathcal{M}^{m} \mathcal{M}^{\prime k}\right)$, by using $\mathcal{M M}=1$ we get

$$
\operatorname{Tr}\left(\mathcal{M} \mathcal{M}^{\prime k}\right)=-\operatorname{Tr}\left(\mathcal{M} \mathcal{M}^{\prime k}\right)=0
$$

Finally, due to Eq. (A27), the dilation could be replaced by $\operatorname{Tr} \mathcal{M}^{\prime 2}$. In summary, the higher-order corrections of $\alpha^{\prime}$ are constructed using $\mathcal{M}^{\prime 2}$. For example,

$$
\begin{gathered}
\mathcal{O}\left(\alpha^{\prime}\right): a_{1} \operatorname{Tr} \mathcal{M}^{\prime 4}+a_{2}\left(\operatorname{Tr} \mathcal{M}^{\prime 2}\right)^{2} \\
\mathcal{O}\left(\alpha^{\prime 2}\right): b_{1} \operatorname{Tr} \mathcal{M}^{\prime 6}+b_{2} \operatorname{Tr} \mathcal{M}^{\prime 4} \operatorname{Tr} \mathcal{M}^{\prime 2}+b_{3}\left(\operatorname{Tr} \mathcal{M}^{\prime 2}\right)^{3} \\
\vdots \\
\mathcal{O}\left(\alpha^{\prime k-1}\right): d_{1} \operatorname{Tr} \mathcal{M}^{\prime 2 k}+d_{2} \operatorname{Tr} \mathcal{M}^{\prime 2 k-2} \operatorname{Tr} \mathcal{M}^{\prime 2} \\
+d_{3} \operatorname{Tr} \mathcal{M}^{\prime 2 k-4}\left(\operatorname{Tr} \mathcal{M}^{\prime 2}\right)^{2}+\ldots
\end{gathered}
$$

Furthermore, considering the action at zeroth order [Eq. (A16)], the variation for $g_{x x}$ gives

$$
\delta \bar{I}_{0}=\int d x e^{-\Phi}\left[\Phi^{\prime 2}+\frac{1}{8} \operatorname{Tr}\left(\mathcal{M}^{\prime}\right)^{2}\right] \delta g_{x x}
$$

This variation can be generalized to the higher orders with $X_{2 k}\left(\mathcal{M}^{\prime}\right)=\operatorname{Tr}\left[\left(\mathcal{M}^{\prime}\right)^{2 k_{1}}\right] \cdots \operatorname{Tr}\left[\left(\mathcal{M}^{\prime}\right)^{2 k_{n}}\right], k=k_{1}+k_{n}$ : 


$$
\delta \bar{I}_{k}=\frac{\beta k}{2(4 k-1)} \int d x e^{-\Phi} X_{2 k}\left(\mathcal{M}^{\prime}\right) \operatorname{Tr}\left(\mathcal{M}^{\prime}\right)^{2},
$$

where

$$
\delta g_{x x}=\beta \alpha^{\prime k} X_{2 k}\left(\mathcal{M}^{\prime}\right) .
$$

If we substitute the redefinition (A37) back into Eq. (A35) and set $\frac{\beta k}{2(4 k-1)}=-1$, we find that the terms with $\operatorname{Tr} \mathcal{M}^{\prime 2}$ can be eliminated when we sum Eqs. (A35) and (A36). In other words, we can safely set $\operatorname{Tr} \mathcal{M}^{\prime 2}=0$ for $\alpha^{\prime}$-corrected terms in the action and obtain

$$
\begin{aligned}
\mathcal{O}\left(\alpha^{\prime}\right) & : a_{1} \operatorname{Tr} \mathcal{M}^{\prime 4} \\
\mathcal{O}\left(\alpha^{\prime 2}\right) & : b_{1} \operatorname{Tr} \mathcal{M}^{\prime 6} \\
\vdots & \\
\mathcal{O}\left(\alpha^{\prime k-1}\right) & : d_{1} \operatorname{Tr} \mathcal{M}^{\prime 2 k}+d_{4} \operatorname{Tr} \mathcal{M}^{\prime 2 k-4} \operatorname{Tr} \mathcal{M}^{\prime 4} \ldots
\end{aligned}
$$

The action with higher-order $\alpha^{\prime}$ corrections then reduces to

$$
\begin{aligned}
\bar{I} \equiv & -\int d x e^{-\Phi}\left(-\Phi^{\prime 2}+\sum_{k=1}^{\infty}\left(\alpha^{\prime}\right)^{k-1} \bar{c}_{k} \operatorname{Tr}\left(\mathcal{M}^{\prime 2 k}\right)\right. \\
& + \text { multitraces }) .
\end{aligned}
$$

After extracting the overall minus sign of the action above, the even orders of $\alpha^{\prime}$ corrections acquire a minus sign. Since $\bar{c}_{k}$ is the coefficient of $\operatorname{Tr} \mathcal{M}^{\prime 2 k}$, which is not modified by $\Phi^{\prime k} \simeq \frac{1}{8}\left(\frac{k-1}{k-3}\right) \Phi^{\prime k-2} \operatorname{Tr} \mathcal{M}^{\prime 2}$, the values of $\left|c_{k}\right|$ and $\left|\bar{c}_{k}\right|$ are the same. Moreover, by using Eqs. (A9), (A13), and (A14) to all orders, we find $\bar{c}_{1}=c_{1}=-\frac{1}{8}, \bar{c}_{2 k+1}=c_{2 k+1}$, and $\bar{c}_{2 k}=-c_{2 k}$. It is worth noting that the relationships between $\bar{c}_{k}$ and $c_{k}$ are not changed after including the contributions of the multitrace terms.
[1] G. Obied, H. Ooguri, L. Spodyneiko, and C. Vafa, De Sitter space and the Swampland, arXiv:1806.08362.

[2] P. Agrawal, G. Obied, P. J. Steinhardt, and C. Vafa, On the cosmological implications of the string Swampland, Phys. Lett. B 784, 271 (2018).

[3] S. K. Garg and C. Krishnan, Bounds on slow roll and the de Sitter Swampland, arXiv:1807.05193.

[4] H. Ooguri and C. Vafa, Non-supersymmetric AdS and the Swampland, Adv. Theor. Math. Phys. 21, 1787 (2017).

[5] O. Hohm and B. Zwiebach, Non-perturbative de Sitter vacua via $\alpha^{\prime}$ corrections, arXiv:1905.06583.

[6] O. Hohm and B. Zwiebach, Duality invariant cosmology to all orders in $\alpha^{\prime}$, arXiv:1905.06963.

[7] G. Veneziano, Scale factor duality for classical and quantum strings, Phys. Lett. B 265, 287 (1991).

[8] A. Sen, $\mathrm{O}(\mathrm{d})$ x $\mathrm{O}(\mathrm{d})$ symmetry of the space of cosmological solutions in string theory, scale factor duality and twodimensional black holes, Phys. Lett. B 271, 295 (1991).

[9] A. Sen, Twisted black p-brane solutions in string theory, Phys. Lett. B 274, 34 (1992).

[10] K. A. Meissner and G. Veneziano, Symmetries of cosmological superstring vacua, Phys. Lett. B 267, 33 (1991).
[11] K. A. Meissner, Symmetries of higher order string gravity actions, Phys. Lett. B 392, 298 (1997).

[12] O. Hohm, W. Siegel, and B. Zwiebach, Doubled $\alpha^{\prime}$-geometry, J. High Energy Phys. 02 (2014) 065.

[13] O. Hohm and B. Zwiebach, Double field theory at order $\alpha^{\prime}$, J. High Energy Phys. 11 (2014) 075.

[14] D. Marques and C. A. Nunez, T-duality and $\alpha^{\prime}$-corrections, J. High Energy Phys. 10 (2015) 084.

[15] O. Hohm, Background Independence and Duality Invariance in String Theory, Phys. Rev. Lett. 118, 131601 (2017).

[16] O. Hohm and B. Zwiebach, T-duality constraints on higher derivatives revisited, J. High Energy Phys. 04 (2016) 101.

[17] W. H. Baron, E. Lescano, and D. Marques, The generalized Bergshoeff-de Roo identification, J. High Energy Phys. 11 (2018) 160.

[18] C. Krishnan and de Sitter, $\alpha^{\prime}$-corrections \& duality invariant cosmology, arXiv:1906.09257.

[19] P. Wang, H. Wu, and H. Yang, Correspondence between genus expansion and $\alpha^{\prime}$ expansion in string theory, arXiv: 1703.05217 . 